\title{
Thinking About Thinking and Feeling About Feeling
}

\author{
J. Moore \\ University of Wisconsin-Milwaukee
}

\begin{abstract}
Traditional clinical psychology generally posits "mental" events that differ from "behavioral" events. Mental events are not publicly observable, take place in a different dimension from overt behavior, and are the topic of primary concern. For example, mental events are often taken to be causes of troublesome overt behavior. In addition, the mental events themselves may be regarded as troublesome, independent of their relation to any specific overt behavior. Therapy is usually aimed at fixing these troublesome mental events, under an assumption that improvement in the client's status will follow in due course. Behavior analysis has its own position on the relations among clinical matters, overt behavior, and such private events as thinking and feeling. In a behavior-analytic view, private events are behavioral phenomena rather than mental phenomena. They are not initiating causes of behavior; rather, they are themselves caused by antecedent conditions, but they may contribute to discriminative control over subsequent behavior, both verbal and nonverbal. Verbal processes are viewed as vitally important in understanding troublesome behavior. However, the circumstances that cause both the troublesome private events and the troublesome behavior in the first place still need to be addressed. Finally, clinical behavior analysis will need to market its insights into diagnosis and treatment very adroitly, because it rejects the mentalism upon which most traditional forms of therapy are predicated and the mentalism that most consumers expect to encounter.
\end{abstract}

Key words: behavior analysis, private events, thinking, feeling, methodological behaviorism, stimulus equivalence, relational frame theory

In general terms, the present papers are concerned with how behavior analysts conceive of the relation between clinical matters and private events, such as thinking and feeling. Given that this relation is of immense practical and theoretical significance, it is probably not discussed as often as it should be in the literature of clinical behavior analysis. I suspect that this lack of attention has given rise to the impression that other approaches, such as cognitive behavior therapy, are superior to clinical behavior analysis precisely because they find a place in their assessments and interventions for thinking and feeling, whereas clinical

This article is based on discussants' remarks at the symposium on "Behavior analysts: What do they think about thinking and feeling?" held at the convention of the Association for Behavior Analysis in Chicago, May, 1997. I thank Rob Hawkins for his kind invitation to participate in the symposium.

Correspondence concerning this article should be addressed to J. Moore, Department of Psychology, University of Wisconsin-Milwaukee, Milwaukee, Wisconsin 53201 (E-mail: jcm@ csd.uwm.edu). behavior analysis does not. However popular this impression might be, I contend that the present papers clearly show it to be mistaken. Nevertheless, it stands to reason that if we do begin to talk about private events more and do come to a greater consensus about when, where, and in what ways private events are important in clinical matters, behavior analysts can provide better services for clients and a better overall theoretical understanding of the human condition.

\section{HISTORICAL AND CONCEPTUAL BACKGROUND}

The topics of thinking and feeling play a huge role in most clinical approaches, and concerns about how to meaningfully integrate these topics into a systematic theoretical position are not new. My view of the historical and conceptual background of behavioral concerns with thinking and feeling is roughly as follows. If we were to go back 100 or 125 years, thinking and feeling, rather than behavior as such, 
were of primary concern in the discipline. Behavior was publicly observable, and a given phenomenon had to be publicly observable to count as behavior. Because thinking and feeling were obviously not publicly observable, they were therefore regarded as mental and as ontologically distinct from behavior. Nevertheless, the prevailing view was that thinking and feeling could be dealt with scientifically through introspection. It is important to note that thinking and feeling occurred at just the right temporal location to be regarded as causes of behavior.

As most students of the history of psychology know, Watson's classical stimulus-response (S-R) behaviorism rejected the introspective analysis of subjective experience in a supposed mental dimension. It focused instead on a publicly observable subject matter: behavior. Classical behaviorism did not shrink from traditional topics, but rather tried to analyze them as well as it could, using the concepts and principles it had available. For example, thinking was treated as subvocal speech or other forms of laryngeal habits, and feeling as another form of conditioned response. Particularly critical examples of the latter for Watson were the emotions of fear, rage, and love. In a more general sense, emotions were not something other than behavior, but rather behavior itself, in all its wonder.

Unfortunately, however, psychologists soon began to see that all responses were not correlated with eliciting stimuli in the way that classical behaviorism required. In addition, the S-R model does not easily accommodate how we come to use subjective terms to describe various conditions inside our bodies. Finally, other sciences seemed to be making progress by postulating unobservable phenomena, so why should not psychology? Was psychology not throwing the baby out with the bathwater by restricting itself to publicly observable phenomena? As a consequence of these sorts of questions, Watson's classical S-R behavior- ism with its emphasis on publicly observable factors had begun to lose some of its favor.

Researchers then began to amend the S-R formulation by inserting unobserved "organismic" variables that mediated the relation between stimulus and response. This strategy resulted in the S-O-R formulation of mediational neobehaviorism. The principal question was how to guarantee that the use of the mediating, organismic term was scientifically meaningful and respectable. Although the entire story is quite complicated, suffice it to say at this point that the organismic variables were given the status of "theoretical terms" in the S-O-R model, and then were "operationally defined." An operational definition specified the publicly observable phenomena (i.e., operations) that made it possible to use the term in ways that commanded agreement. Once agreement was secured, the term was acceptably meaningful. However, the term could be given either (a) an intervening variable interpretation, which allowed no surplus meaning beyond its immediate application in an equation or scientific statement; or (b) a hypothetical construct interpretation, which did allow surplus meaning, perhaps even including the "mental" as an ontology that differed from the physical. In some instances, the theoretical terms were intervening variables, and in others they were hypothetical constructs. When theorists employed intervening variables, their position was essentially that of one or another version of philosophical or logical behaviorism. Thinking and feeling were regarded as "dispositional." However, when theorists employed hypothetical constructs, and most did, usually because of the greater degrees of freedom in theory construction, then their position was that of the mature form of "methodological behaviorism" (Moore, 1981, 1995b, 1996).

Again, it is important to note that the publicly observable measures taken to operationally define the hypothetical 
construct were a license for speaking meaningfully of the unobserved theoretical entities. The measures were evidence, rather than comments on the ontology of the hypothetical construct. The ontology of the hypothetical construct was typically that of the mental. Indeed, the whole methodological behaviorist approach of treating private phenomena as hypothetical constructs virtually guaranteed that they would be regarded as mental causes of behavior, and because methodological behaviorism remains influential, such private phenomena as thinking and feeling continue to be regarded as mental causes in virtually all of contemporary psychology. Clinical psychology is especially vulnerable to such influences because of charges that it deals with a subject matter that cannot be studied "scientifically."

The pervasiveness of the medical model in traditional analyses of abnormality is additional testimony to the power of this approach. The medical model is a general orientation to the problem of abnormality in which bizarre, extreme, and disturbing behaviors are viewed as symptoms caused by underlying pathological private events, such as pathological processes of thinking and feeling, in the same way that cough, fever, and sore throat are viewed as symptoms caused by an underlying medical pathology, such as bacteria or virus. In each case, the task of the specialist is to infer the nature of the underlying "disease" or "pathology" and the underlying "cause" on the basis of the evidence provided by the "symptoms." Appeal to the causal efficacy of private or mental entities, such as disturbed thinking and feeling, in medical model approaches of traditional clinical psychology is made legitimate by the practices of mentalism and methodological behaviorism, especially operational definitions in the sense reviewed above.

One can further point out that various forms of therapy have evolved that are concerned with changing feelings largely through verbal processes. The orthodox assumption is that by using verbal behavior, one can bring about changes in mental phenomena, including thoughts and feelings, from which any changes in behavior would naturally follow. One can note the ascendance and continuing dominance of the "talking therapies" in this regard. All this is a consequence of methodological behaviorism as well. Moreover, the whole approach flourishes in traditional behavior therapy as well as the talking therapies. In sum, therapists of a wide variety of different persuasions believe they are being "scientific" about their approach, whereas in reality they are doing the same old thing, just renamed, all as a consequence of the commitment to methodological behaviorism and the accompanying mentalistic view of how to engage the relation between clinical matters and private events.

\section{BEHAVIOR ANALYSIS AND PRIVATE EVENTS}

As Anderson, Hawkins, Freeman, and Scotti (2000) note, behavior analysis has a different perspective on the relation between clinical matters and private events. Indeed, the behavioranalytic position on private events has always struck me as one of its strongest features. Rather than regard private phenomena as something other than behavior, something that had to be pursued either by rational philosophical inquiry or by introspection or as a hypothetical construct, behavior analysis regards private events as formally and explicitly behavioral. Two issues that distinguish the behavior-analytic position from the traditional position are especially important. The first is the ontology of private events. The second is their causal mode.

\section{Ontology of Private Events}

Often, behavior analysis seems to sidestep questions of ontology: "It is a little too simple to paraphrase the behavioristic alternative by saying that there is indeed only one world and that 
it is the world of matter, for the word 'matter' is then no longer useful" (Skinner, 1969, p. 248). However, behavior analysis certainly adopts a physical, materialist position: "Private and public events have the same kinds of physical dimensions" (Skinner, 1969, p. 228); "A radical behaviorism denies the existence of a mental world" (Skinner, 1969, p. 267); "No special kind of mind stuff is assumed" (Skinner, 1974 , p. 220); "I am a radical behaviorist simply in the sense that I find no place in the formulation for anything which is mental" (Skinner, 1964, p. 106). Behavior analysis recognizes that most of the variables with respect to which the human organism behaves are publicly observable. However, not all the relevant variables are publicly observable. Private phenomena, accessible only to one individual, may be important in the control of behavior. Nevertheless, they need not be approached as theoretical inferences about causal phenomena from another dimension, such as the "mental" dimension, simply because they are not accessible to more than one person. Talk of the mental is attributable to longstanding preconceptions about the nature of human beings arising from social, cultural, and theological sources, rather than from any observational basis. Skinner (1953) addressed the matter as follows:

When we say that behavior is a function of the environment, the term "environment" presumably means any event in the universe affecting the organism. But part of the universe is enclosed within the organism's own skin. Some independent variables may, therefore, be related to behavior in a unique way. ... With respect to each individual, in other words, a small part of the universe is private.

We need not suppose that events which take place within an organism's skin have special properties for that reason. A private event may be distinguished by its limited accessibility but not, so far as we know, by any special structure or nature. (Skinner, 1953, pp. 257-258)

Behavior analysis incorporates private phenomena in the same behavioral dimension as public phenomena. As reviewed elsewhere (Moore, 1980), some private phenomena are felt conditions of the body (e.g., aches, pains, feelings, and emotions), whereas others are covert forms of behavior (e.g., thinking, problem solving, recalling, and imagining). Consideration of private events means that radical behaviorists can say quite legitimately that they "do not believe there is a world of mentation or subjective experience that is being, or must be ignored" (Skinner, 1978, p. 124; for additional discussion of mental and cognitive terms, see Skinner, 1989, 1990).

With respect to covert forms of behavior, Skinner commented on the ontology of thinking in several places. Here are two representative passages:

The simplest and most satisfactory view is that thought is simply behavior-verbal or nonverbal, covert or overt. It is not some mysterious process responsible for behavior but the very behavior itself in all the complexity of its controlling relations, with respect to both man the behaver and the environment in which he lives... . So conceived, thought is not a mystical cause or precursor of action, or an inaccessible ritual, but action itself, subject to analysis with the concepts and techniques of the natural sciences, and ultimately to be accounted for in terms of controlling variables. (Skinner, 1957, p. 449)

Usually, however, the term [thinking] refers to completed behavior which occurs on a scale so small that it cannot be detected by others. Such behavior is called covert. The commonest examples are verbal, because verbal behavior required no environmental support and because, as both speaker and listener, a person can talk to himself effectively; but nonverbal behavior may also be covert. Thus, what a chess player has in mind may be other moves he has made as he has played the game covertly to test the consequences. ... Covert behavior is almost always acquired in overt form and no one has ever shown that the covert form achieves anything which is out of reach of the overt. Covert behavior is also easily observed and by no means unimportant, and it was a mistake for methodological behaviorism and certain versions of logical positivism and structuralism to neglect it simply because it was not "objective." ... It does not explain overt behavior: it is simply more behavior to be explained.

The present argument is this: mental life and the world in which it is lived are fictions. They have been invented on the analogy of external behavior occurring under external contingencies. Thinking is behaving. The mistake is in allocating the behavior to the mind. (Skinner, 1974, pp. 106-107) 


\section{Causal Mode}

Further important questions about private events concern their causal mode (Moore, 1980, 1992, 1995a). In a behavior-analytic view, private phenomena do not cause behavior in the sense that the inferred entities of mediational neobehaviorism are presumed to cause behavior. Private phenomena are simply part of the environmental context in which behavior occurs (Hayes \& Brownstein, 1986). As Anderson et al. (2000) point out, they are not always present. Even when they are present, they do not always influence behavior. When they are present and do influence behavior, some circumstances are responsible for their doing so.

Consider the causal status of the covert activity of thinking. As Skinner (1953) noted,

The private event is at best no more than a link in a causal chain, and it is usually not even that. We may think before we act in the sense that we may behave covertly before we behave overtly, but our action is not an "expression" of the covert response or a consequence of it. The two are simply attributable to the same variables. (p. 279)

Again, behavior analysis is here distinguishing itself from traditional approaches. Behavior analysis accepts thinking, not as mental but as behavioral, and does not give thinking or any other covert activity an initiating causal power. At the very least, one has to specify where the thinking comes from, and why the thinking then exerts an influence.

For behavior analysis, the causal mode of covert activity is that of discriminative control. However, this discriminative control is the result of a certain developmental process. If it were not, the behavior-analytic approach would not be appreciably different from the traditional approach. Skinner was always sensitive to maintaining this difference. For example, covert behavior may come to exert control by virtue of the stimulus control shared between public and private stimuli as a response is executed. Sup- pose an individual engages in some temporally segmented form of behavior that has both overt and covert components, as in solving a problem. Ordinarily, the overt components will exert stimulus control during the process. However, the covert components will acquire some measure of stimulus control because they are present as well. In the future, if the public stimuli that occasion the overt response are inadequate (e.g., by being too weak), the individual may engage in the covert behavior and still solve the problem. That is, something goes on covertly that is a component of that which goes on overtly when the act is ordinarily carried out. The stimulus control is exerted via the private components, through interoceptive and proprioceptive systems.

In many cases, covert behaviors are acquired in their overt form. The behavior then recedes to the private form where, as private stimulation, it then joins with other stimuli to form a complex of controlling stimuli. Such control is by no means inevitable, any more than control by a given public stimulus is inevitable. Again, the control exerted by this verbal behavior does not differ from that which would develop if the same verbal behavior arose as a public event.

Why should public behavior recede to the covert form (Skinner, 1957, pp. 434ff.)? One possibility is that the public form is punished (Hyten \& Chase, 1991). Individuals are often encouraged to read silently when they are bothersome to others around them. A second possibility is that the public environment contains only some portion of the discriminative stimuli that ordinarily occasion the response in its public form, thereby making the behavior weak. A third possibility is that the behavior is faster and less troublesome in a covert form, particularly when the behavior is in its inchoate or incipient stages. A common example involving all three processes is when individuals attempt to solve a difficult problem. In a public setting, they might try to pri- 
vately solve the problem "in their heads." However, when they are alone, the accompanying verbal behavior might reemerge in an overt form, and they might begin to "talk to themselves out loud" as they attempt to solve the problem.

Still to be discussed in regard to the question of causal mode is the topic of behavior-behavior relations. That is, behavior analysts ordinarily analyze events in terms of behavior-environment relations, granting that part of the environment may be within the skin. Thinking is actually said to be covert operant behavior that exerts discriminative control. What are the implications of this approach?

To be sure, behavior does have stimulus consequences. One can leave aside the discussion of the straightforward consequences such as reinforcement or punishment. People contact the behavior of another individual through a standard modality, such as vision, audition, or touch. Behavior analysts can legitimately say that the behavior of one individual is discriminative for some subsequent behavior of another individual. Behavior analysts also can say that one instance of an individual's behavior is discriminative for a subsequent instance of that individual's behavior, as when engaging in a sequence of behavior like problem solving. Behavior analysts would presumably want to specify the medium of contact. It may well be visual or auditory. It might also be through interoception or proprioception, as noted earlier, in cases in which the response is covert.

However, when the response is covert, the additional factor of the "problem of privacy" applies. Skinner began to discuss this problem in 1945, and continued in 1953,1957, 1964, and 1974. The problem of privacy means that the verbal community may not be able to provide the appropriate differential reinforcement that brings responses under the control of private stimuli. The verbal community does not have direct access to the private stimuli, and must resort to other factors, with the result that the presentation or withholding of reinforcement may not be correlated with the presence or absence of the private stimulus. As a result, a wide variety of incidental or extraneous stimuli may influence any responses that individuals make ostensibly on the basis of their private stimuli. If incidental or extraneous stimuli influence these responses, the control the responses exert over subsequent behavior may be less effective than the control that public stimuli exert.

Let me return to the matter of behavior-behavior analyses. This form of analysis is acceptable, provided one aspect of the behavior can be linked to the environment, which is the point at which effective action can be taken. Traditional analyses, not only of thinking but also of feelings and emotions, typically do not do this. They mistakenly accept what is essentially one form of behavior and use it as an initiating cause for a succeeding form, making a behavior-behavior analysis that does not lend itself to prediction and control. When behavior analysis links one aspect of the behavior to the environment, as it does when it characterizes thinking as behavioral, but then allows thinking to be a covert activity that has discriminative effects, behavior analysis does not make the same kind of behavior-behavior analyses as does traditional psychology. It is internally consistent because it establishes the functional interrelation between behavior and environmental circumstances, and it avoids the mentalism of initiating inner causes (Hayes \& Brownstein, 1986).

Consider next the topic of feelings. What causes feelings? Presumably, what one feels are conditions of the body created by contact with contingencies. An answer to a question of the origin of feelings is presumably to be found in an analysis of the environmental circumstances with which an organism is in contact. Any sense of "epiphenomenal" noted in the present 
discussion is the sense of whether one goes back far enough in the causal chain. If emotions are only the middle link, then presumably the causal analysis of a behavioral event needs to focus on the earliest link possible.

What then is the relation between feelings and behavior? In a behavioranalytic view, contingencies, not feelings, cause behavior. The feelings are collateral by-products of the causal process. Even if feelings are cited as causes of behavior, one needs to ask what causes the feelings, and the answer is to be found in the prevailing environmental circumstances.

Presumably, one changes feelings by changing the contingencies that cause the conditions one feels. As noted earlier, feelings are not causal for behavior in the sense of initiating internal entities, nor are they causal in the sense of the mediating phenomena of neobehaviorism (e.g., the influential two-process approach of Rescorla \& Solomon, 1967, p. 178: "the concomitance we do observe between CRs and instrumental responding is mediated by a common central [emotional] state, and the changes in that state are subject to the laws of Pavlovian conditioning").

\section{THE ESTABLISHING OPERATION}

An important feature of an analysis in terms of the relation between environment and behavior, of course, is the establishing operation, as Dougher and Hackbert (2000) point out. Some examples of common emotions and feelings may help to illustrate the point. Anxiety may be characterized as bodily conditions created by inconsistent contingencies, perhaps involving impending aversive or punishing stimuli or impending withdrawal of positive reinforcement. Guilt might be characterized as bodily conditions created by social punishment or loss of social reinforcement. Depression might be characterized as bodily conditions created by extinction or other loss of reinforcement, often unpredicted and in- consistent, in which an individual's repertoire is not adequate to create conditions that promote reinstatement of reinforcement. All of these constitute establishing conditions that make more probable certain classes of behavior.

I am not sure I fully understand Dougher and Hackbert's statement that "Many ... [clinical] observations, however, are not readily explainable in terms of the three-term contingency" (p. 13). If they are suggesting that some behavior develops and comes under verbal stimulus control even though a response is not directly reinforced in the presence of a particular verbal discriminative stimulus, I readily agree. The phenomenon known generically as "stimulus equivalence" is surely testimony to this effect. Nevertheless, I continue to believe that the contingency is the appropriate analytical unit. Findings such as stimulus equivalence indicate that it may prove to be necessary to expand existing conceptions of how an antecedent stimulus that participates in a contingency acquires its discriminative function, rather than to discard altogether the notion of the contingency as the unit of analysis. Relational frame theory (Hayes, 1994; Wilson \& Hayes, 2000) is of course an exceedingly promising possibility for understanding how a verbal stimulus acquires its discriminative function. In any event, I contend that behavior analysis is a historical science, and presumably the "meaning" of discriminative stimuli and conditioned reinforcers comes from their past involvement in contingencies. I think clinical behavior analysis $r e$ quires the exploration of historical variables when it comes to explaining human behavior. Whether any particular behavior analyst does it well enough is another question, of course, and I suspect that what separates effective therapists from ineffective ones is the degree to which they effectively explore historical variables.

In this regard, I once heard it said in connection with behavior therapy that "One doesn't need to know how the 
fire started in order to put it out." My guess is that this saying arose in the early days of behavior therapy. At that time, behavior therapists were eager to establish the validity of their approach. To do so, they sought to distinguish themselves from those who practiced other approaches, such as psychoanalysts, who argued that the only way to help the client was to look for the underlying cause of a behavioral disorder deep in the personality structure of the client. In the Freudian view, for example, the personality structure was presumed to malfunction in many instances because of aberrant psychosexual episodes during the client's youth.

It may well be that therapists need not look for the cause in terms of Freudian processes, but that is because Freudian processes do not exist, not because looking for the cause of the troublesome behavior in the history of the client's interactions with the environment is without merit. It strikes me as entirely wrong to believe that one can safely neglect the origin of a behavioral disorder and still provide a service to the client. The implication that one can safely neglect the origin strikes me as the legacy of Watsonian and Hullian mechanical models of behavioral disorders, rather than behavior analysis. At the very least, given what is known about the conditionality of behavior, therapists would want to know the cause of the troublesome behavior. That is, therapists would want to know the establishing conditions and contingencies that are responsible for the troublesome behavior, so that they can design interventions concerned precisely with those establishing conditions and contingencies as they exist in the client's life outside the therapeutic intervention. They can then prevent those contingencies from having the same troublesome effect the next time the client encounters them, just as one can prevent a fire from reoccurring if one knows how the fire started.

\section{THE IMPORTANCE OF VERBAL PROCESSES}

All this implies that verbal processes do matter in clinical behavior analysis. The conditions felt are discriminative for verbal labels. The verbal labels may in turn exert discriminative control over subsequent behavior, both verbal and nonverbal. Any verbal behavior so occasioned may come to be discriminative for further behavior, and so on. Presumably, the process of stimulus generalization is also involved. The resulting behavioral processes are often persistent, and are perhaps related to what is called self-defeating behavior or self-fulfilling prophecies. The verbal stimuli may also function as conditioned stimuli that evoke their own conditions felt and that interact with any other behavior effects, perhaps even as conditioned establishing stimuli (Schlinger \& Blakely, 1987).

An important issue is the extent to which the phenomenon of stimulus equivalence is involved. Wilson and Hayes (2000) make an important point when they suggest that there is no indication that an animal's report of an aversive event, such as pecking a key to report that it just received a shock, is itself aversive. However, a human's verbal report of an aversive event, such as talking about an unpleasant experience, is presumably aversive. Indeed, it may be the basis for phobias, panic attacks, and a wide variety of other unfortunate states of affairs. As Wilson and Hayes mention, the relation is bidirectional. Why should this be the case? The link is that the effects in humans may be verbally mediated, as illustrated in the principle of symmetry. That is, just as experiencing an aversive event directly is aversive, so are the words used in conjunction with the same event aversive. If so, then new avenues are opened to an understanding of the origin and spread of troublesome conditions, and the vehicle that travels those avenues is verbal behavior.

Wilson and Hayes (2000) forcefully 
present their version of the importance of these verbal processes. Verbal cues, of course, are caused by a set of contingencies associated ultimately with the verbal community. An important issue is how these verbal cues come to exert their subsequent regulatory effect (i.e., discriminative control) over subsequent activity. Wilson and Hayes suggest that Skinner's approach cannot account for the control. It seems to me that Skinner's approach acknowledges the fact that such control would arise. For example, Skinner (1953) states that an individual "controls himself precisely as he would control the behavior of anyone else-through the manipulation of variables of which behavior is a function" (p. 228). Presumably, selfgenerated verbal stimuli are among the variables that can be manipulated in self-management and self-control. However, whether Skinner's approach can account for the principle by which the regulatory control arises is a different manner. This principle has to do with the very definition of verbal behavior, particularly with regard to the role of the listener in verbal behavior. For example, one might say that a verbal event is one in which the listener participates in arbitrarily applicable relational responding, or derived relational responding, or coordinated frames of reference. Skinner's approach is not nearly so broad, as Wilson and Hayes point out. If the speaker and listener are the same individual, as in cases of self-control or self-attributions or self-defeating prophecies or whatever else a client might present at an intake interview, the tremendous spread of the response, whether public conduct or private feeling, needs to be recognized. Again, the question is the principle by which the behavioral effects spread to the new circumstances. Clearly, the individual does not need to be exposed directly to the new circumstances for the response to spread to them. Skinner simply did not address these matters, whereas others such as Wilson and Hayes do. Whether all this theoretical activity leads to a new con- ception of verbal behavior, as Leigland (1997) has recently discussed, remains to be determined. In any case, I look forward to its resolution, because it is probably the single biggest theoretical issue in contemporary behavior analysis.

\section{BEHAVIOR ANALYSIS AND THERAPY}

What then are the implications of the behavior-analytic worldview for therapy? Debates rage in traditional psychology about whether the success of therapy should be judged in terms of whether it creates insight, changes behavior, or changes feelings that may (or may not) be related to changes in overt behavior. Debates also rage about who is to judge the success: client, therapist, or third party. In a behavioranalytic view, feelings are behavior. They are private respondents, as opposed to the public operants that most people regard as behavior, so in an important sense a dichotomy that sets feelings against behavior is false. Even with the traditional dichotomy, I suggest that a therapy that does not change at least some aspect of behavior is likely to be judged as ineffective. The question is how efficiently one is going to change behavior in the desired ways. If one followed a client around all day for 30 or 60 days with an arsenal of appropriate positive reinforcers, one could presumably change behavior quite a bit. Let's see-16 hours a day for 60 days at, say, $\$ 135$ per hour for the first 8 hours a day, $\$ 202$ for the next 4 , and $\$ 270$ for the next 4 . Think the HMO would go for it? Realistically, therapy is going to be carried out at least partly through verbal processes, even by clinical behavior analysts. The verbal processes are going to focus on removing the kind of maladaptive verbal control described above and preventing maladaptive generalization or equivalence effects. Presumably, that is the basis by which cognitive and talking therapies achieve their effects, 
when they do, although they do not identify it as such.

However, even though changing verbal behavior to correct unwanted generalization and maladaptive equivalence relations is an important component of therapy, it seems to me that they do not exhaust all the techniques that need to be applied. One still has to deal with the environmental conditions that cause the feelings in the first place. The client needs to be provided with a repertoire that is adequate to deal with the loss of reinforcement (e.g., in the case of depression), to avert maladaptive avoidance responses (e.g, in the case of phobias or anxiety attacks), or whatever else. As important as understanding verbal processes proves to be, and Wilson and Hayes (2000) are very persuasive on this matter, it seems to me that therapists must remain aware that the circumstances that gave rise to the problem also need to be remedied. These steps involve constructive concern with the actual nonverbal repertoires of clients. I am sure that an important component of this process will prove to be verbal, as in getting a phobic individual to discuss riding in an automobile after a terrible wreck. However, if an individual is depressed because of ineffective interpersonal relationships, he or she is going to keep getting depressed unless he or she develops a new and more effective repertoire of dealing with others. Developing such a repertoire strikes me as a matter of direct contingencies, certainly social, although not necessarily verbal in the sense of equivalence classes and relational framing.

Some of these circumstances may change while the therapeutic intervention is taking place, but will change independently of that intervention. This state of affairs is called spontaneous remission. Similarly, the circumstances that originally caused the condition felt are often remote, and may have to be dealt with at least initially via verbal processes. Instructions and advice exert discriminative control over behav- ior, resulting in different modes of contact with the environment. Shaping and equivalence classes might also be involved.

To the extent that different forms of therapy have proved successful in dealing with clinical problems, they have presumably involved different ways of bringing clients into contact with verbal stimuli. These forms of therapy may be differentially successful based on the characteristics of the client and on how susceptible the client is to verbal control. Thus, techniques of clientcentered therapy, humanistic therapy, Freudian psychoanalysis, rational-emotive therapy, transactional analysis, and so forth, may be successful or not, depending on how well the characteristics of the client, such as sensitivity to verbal stimuli, intersect with the characteristics of the therapist, such as skill in presenting verbal stimuli appropriate to the status of the client. The mistake is in not understanding the processes by which the form of therapy exerts its therapeutic effect, and in attributing it to changes in the personality structure or other entities from a mental dimension.

\section{SUMMARY AND CONCLUSIONS}

In summary, then, the fundamental concern of behavior analysts is with contingencies. Thinking is behavior that is generated by one set of contingencies and then enters into another set of contingencies that affect subsequent behavior. Thinking is not an initiating mental activity, although it may participate in the discriminative control over future behavior. Emotions are conditions felt as a result of contact with various contingencies. In this view, feelings are not initiating mental entities, although they may be discriminative for verbal labels, which can in turn evoke other emotional responses. The verbal labels so engendered may also function as establishing events, as well as discriminative stimuli, for other forms of subsequent behavior. 
As suggested above, verbal processes are important. They are basically how talking therapies achieve their therapeutic benefit. One can question whether talking therapies are the most efficient way to achieve these benefits, but not that they achieve them at least some of the time. As a behavior analyst, I believe that these processes can be sorted out and an even greater benefit can be achieved than by traditional forms of therapy.

I believe that behavior analysis can make a contribution, but behavior analysts also need to be aware of how they present themselves to the world. The behavior-analytic way of approaching these problems is literally the reverse of the way the traditional view approaches them. It will not be readily understood. Considerable shaping of the audience will be necessary, and behavior analysts will have to be ever aware of audience control.

In particular, behavior analysis must avoid the fallacy of the better mousetrap; that is, the popular maxim is that if you build a better mousetrap, the world will beat a path to your door. As much as such maxims might inspire entrepreneurial vigor, I wonder whether this one is entirely accurate. In particular, I wonder whether the world really will beat a path to the door of behavior analysis and proclaim that behavior analysis was essentially correct all along, if behavior analysis really does develop a better therapy.

Consider the arrangement of keys on a typewriter keyboard (Gould, 1991). The predominant layout is called the QWERTY layout. This layout was designed in the early 1870 s, in the era of mechanical linkages on typewriter keys. A common problem during this era was that rapid typing caused the keys to jam. The QWERTY layout was explicitly designed to be suboptimal: It assigned common letters to weak fingers or to those requiring a long reach from the home position, slowing down the maximal speed of typing and thereby preventing jamming of the keys. In fact, research has shown that a layout called the Dvorak layout is superior to the QWERTY layout. Nevertheless, in these days computers and electronic typewriters do not have mechanical linkages to the keys, and they are not in any imminent danger of jamming. Even so, most of us continue to use the older QWERTY layout, perhaps because it was the one on which we learned when mechanical linkages were in common use, even though we could type faster and make fewer errors if we used the Dvorak layout. Dvorak developed a better mousetrap, and the data are all on his side, but the world still has not beaten a path to his door. The implication is clear: Behavior analysts may well be developing a more effective form of therapy, and the data may be all on our side, but the world may still not beat a path to our door. Much more than just data is involved in a decision to embrace a form of therapy as effective.

\section{REFERENCES}

Anderson, C. M., Hawkins, R. P., Freeman, K. A., \& Scotti, J. R. (2000). Private events: Do they belong in a science of human behavior? The Behavior Analyst, 23, 1-10.

Dougher, M. J., \& Hackbert, L. (2000). Establishing operations, cognition, and emotion. The Behavior Analyst, 23, 11-24.

Gould, S. J. (1991). The Panda's thumb of technology. In S. J. Gould, Bully for brontosaurus (pp. 59-75). New York: Norton.

Hayes, S. (1994). Relational frame theory as a behavioral approach to verbal events. In S. C. Hayes, L. J. Hayes, M. Sato, \& K. Ono (Eds.), Behavior analysis of language and cognition (pp. 9-30). Reno, NV: Context Press.

Hayes, S., \& Brownstein, A. (1986). Mentalism, behavior-behavior relations, and a behavior-analytic view of the purpose of science. The Behavior Analyst, 9, 175-190.

Hyten, C., \& Chase, P. N. (1991). Self-editing. In L. J. Hayes \& P. N. Chase (Eds.), Dialogues on verbal behavior (pp. 67-81). Reno, NV: Context Press.

Leigland, S. (1997). Is a new definition of verbal behavior necessary in light of derived relational responding? The Behavior Analyst, 20, 3-9.

Moore, J. (1980). On behaviorism and private events. Psychological Record, 30, 459-475.

Moore, J. (1981). On mentalism, methodological behaviorism, and radical behaviorism. Behaviorism, 9, 55-77.

Moore, J. (1992). On private events and theo- 
retical terms. Journal of Mind and Behavior, 13, 329-346.

Moore, J. (1995a). Radical behaviorism and the subjective-objective distinction. The Behavior Analyst, 18, 33-49.

Moore, J. (1995b). Some historical and conceptual relations among logical positivism, behaviorism, and cognitive psychology. In J. T. Todd \& E. K. Morris (Eds.), Modern perspectives on B. F. Skinner and contemporary behaviorism (pp. 51-74). Westport, CT: Greenwood Press.

Moore, J. (1996). On the relation between behaviorism and cognitive psychology. Journal of Mind and Behavior, 17, 345-368.

Rescorla, R., \& Solomon, R. (1967). Two-process learning theory: Relationships between Pavlovian conditioning and instrumental learning. Psychological Review, 74, 151-182.

Schlinger, H., \& Blakely, E. (1987). Functionaltering effects of contingency-specifying stimuli. The Behavior Analyst, 10, 41-45.

Skinner, B. F. (1945). The operational analysis of psychological terms. Psychological Review, 52, 270-277, 291-294.
Skinner, B. F. (1953). Science and human behavior. New York: Macmillan.

Skinner, B. F. (1957). Verbal behavior. New York: Appleton-Century-Crofts.

Skinner, B. F. (1964). Behaviorism at fifty. In T. W. Wann (Ed.), Behaviorism and phenomenology (pp. 79-97). Chicago: University of Chicago Press.

Skinner, B. F. (1969). Contingencies of reinforcement. New York: Appleton-CenturyCrofts.

Skinner, B. F. (1974). About behaviorism. New York: Knopf.

Skinner, B. F. (1978). Reflections on behaviorism and society. Englewood Cliffs, NJ: Prentice Hall.

Skinner, B. F. (1989). The origins of cognitive thought. American Psychologist, 44, 13-18.

Skinner, B. F. (1990). Can psychology be a science of mind? American Psychologist, 45, 1206-1210.

Wilson, K. G., \& Hayes, S. C. (2000). Why it is crucial to understand thinking and feeling: An analysis and application to drug abuse. The Behavior Analyst, 23, 25-43. 